\title{
Effect Of Gamma Irradiation On Structural, Morphological And Optical Properties Of P-Quaterphenyl Thin Films
}

\author{
A. Attia ${ }^{a, *}$, M. M. Saadeldin ${ }^{b}$, K. Sawaby ${ }^{b}$ \\ ${ }^{a}$ Physics Department, Faculty of Education, Ain Shams University, Roxy, Cairo 11341, Egypt \\ ${ }^{b}$ Physics Department, Faculty of Science, Cairo University, Giza 12613, Egypt \\ attia05@yahoo.com
}

\begin{abstract}
Para-quaterphenyl thin films were deposited onto glass and quartz substrates by thermal evaporation method. p-quaterphenyl thin films were exposed to gamma radiation of Cobat-60 radioactive source at room temperature with a dose of $50 \mathrm{kGy}$ to study the effect of $y$-irradiation on the structure and the surface morphology as well as the optical properties of the prepared films. The crystalline structure and the surface morphology of the as-deposited and $\gamma$-irradiated films were examined using the $\mathrm{X}$-ray diffraction and the field emission scanning electron microscope. The optical constants ( $n \& k$ ) of the as-deposited and $\gamma$-irradiated films were obtained using the transmittance and reflectance measurements, in the wavelength range starting from 250 up to $2500 \mathrm{~nm}$. The analysis of the absorption coefficient data revealed an allowed direct transition with optical band gap of $2.2 \mathrm{eV}$ for the as-deposited films, which decreased to $2.06 \mathrm{eV}$ after exposing film to gamma irradiation. It was observed that the Urbach energy values change inversely with the values of the optical band gap. The dispersion of the refractive index was interpreted using the single oscillator model. The nonlinear absorption coefficient spectra for the as-deposited and $\gamma$-irradiated $p$-quaterphenyl thin films were obtained using the linear refractive index.
\end{abstract}

\section{Keywords:}

p-quaterphenyl; Thin films; Gamma irradiation; Structural properties; Optical properties

Date of Publication: 30-08-2018

DOI: 10.24297/jap.v14i2.7549

ISSN: 2347-3487

Volume: 14 Issue: 2

Journal: Journal of Advances in Physics

Website: https://cirworld.com

This work is licensed under a Creative Commons Attribution 4.0 International License. 


\section{Introduction}

Conjugated organic materials, which have semiconductor properties, have ended up to be promising competitors for different applications in optoelectronics and molecular electronics [1-7]. Owing to their elasticity, ease of production, and low cost, the organic materials are qualified to replace inorganic semiconductors in a numerous of applications (e.g., flat panel displays, solar energy conversion, and plastic integrated circuits). Then, the investigation of these compounds is very important to know about the behavior of their electronic physical properties under different conditions such as exposure to ionizing radiations and changes in temperature, frequency, pressure, ambient gases, etc. Oligomers are one of the organic materials which have been effectively used as active materials as a part of organic transistors [8] or organic lightemitting diodes [9]. These oligomers may be of utility due to their essentially high thermal stability and potential chemical compatibility with the processing required for integrated organic devices. p-quaterphenyl, p-quinquephenyl and p-sexiphenyl are aromatic molecules of the oligophenyls. These oligophenyls have a wide potential as materials for organic transistors [8]. Thin films of these materials are utilized in several practical applications (e.g., laser dye and light blue emitter diode) [10]. Previously, the same authors have studied the characteristics of (Au/p-quaterphenyl film/p-Si/ Al) photodiode using different laser sources [11]. Preparing of $p$-quaterphenyl thin films at room temperature show a high tendency to be in crystalline form. The absorption coefficient data analysis of $p$-quaterphenyl thin films revealed an allowed direct transition with optical energy gap value of $2.35 \mathrm{eV}$ decreasing to $2.05 \mathrm{eV}$ by annealing process [12]. The exposure of the solid materials to gamma radiation gives rise to changes in its microstructural properties and hence the optical and other physical properties are affected [13-15]. Many efforts have been made to study the effect of gamma radiation on thin films and thin film structures of different metal oxides and polymers to detect the suitability of using these films as gamma radiation dosimeters [16-19]. In the present work, we have studied the influence of gamma irradiation on the structure and the surface morphology as well as the optical properties of p-quaterphenyl thin films prepared by thermal deposition technique.

\section{Experimental details}

The p-quaterphenyl $\left(\mathrm{C}_{24} \mathrm{H}_{18}\right)$ powder used in this study was purchased from Aldrich company with purity $98 \%$. Thin films of $p$-quaterphenyl were deposited by a conventional thermal evaporation method using a high vacuum coating unit (Edwards Model E $306 \mathrm{~A}$ ), under a vacuum of $10^{-4} \mathrm{~Pa}$. The quartz crystal thickness monitor (FTM4, Edwards) was employed to monitor the film thickness and the deposition rate as well. The thickness of the film was checked using the Tolansky's interferometric method [20]. Thin films with thickness of $1148 \mathrm{~nm}$ were prepared onto clean glass and quartz substrates for the investigation of structural and optical properties respectively. p-quaterphenyl thin films were irradiated at room temperature, by $50 \mathrm{kGy}$ dose of Cobalt-60 gamma radiation at a dose rate of $6.316 \mathrm{kGy} / \mathrm{h}$ using an irradiation cell, which is available at the National Center for Radiation Research and Technology (NCRRT), Atomic Energy Authority (AEA), Cairo, Egypt. A cooling system was used to avoid the samples heating during the irradiation process. The structural characteristics of the irradiated and unirradiated $p$-quaterphenyl thin films were investigated by using $X$-ray diffractometer (Philips, model $X^{\prime}$ pert) with the characteristic $\mathrm{Cu} \mathrm{K} \mathrm{K}_{\alpha}$ radiation $(\lambda=1.5418 \AA)$. Also, the morphology as well as the particle size of the investigated samples was checked out by scanning electron microscope SEM (Model Quanta 250 FEG; Field Emission Gun). JASCO double-beam spectrophotometer, (Model V 570 UV-Vis-NIR) was utilized to measure the transmittance $(T)$ and reflectance $(R)$ of p-quaterphenyl thin films at normal incidence in the wavelength range $250-2500 \mathrm{~nm}$. The refractive index, $\mathrm{n}$, and the absorption index, k, were estimated using a computer program with the help of the following relations $[21$, 22]:

$\alpha=\frac{1}{d} \ln \left[\frac{(1-R)^{2}}{2 T}+\sqrt{\frac{(1-R)^{4}}{4 T^{2}}+R^{2}}\right]$

where $\alpha$ is the absorption coefficient and $d$ is the thickness of the film.

Then, $k$ is given by the equation: 
Using Eq. (2), $\mathrm{n}$ is calculated as:

$$
\mathrm{n}=\frac{1+\mathrm{R}}{1-\mathrm{R}}+\sqrt{\frac{4 \mathrm{R}}{(1-\mathrm{R})^{2}}-\mathrm{k}^{2}}
$$

Considering that the experimental errors as: $\pm 3 \%$ for $d$ estimation and $\pm 1 \%$ for $T$ and $R$ computation. Then, the errors for $n$ and $k$ were $\pm 3 \%$ and $\pm 2.5 \%$ respectively.

\section{Results and discussion}

\subsection{Structural investigations}

The pattern of X-ray diffraction (XRD) for the powder of p-quaterphenyl in $2 \theta$ angle range $\left(5-50^{\circ}\right)$ is shown in Fig. 1. Miller indices, hkl, and lattice spacing, $d_{h k l}$ corresponding to every diffraction peak and the unit cell parameters of p-quaterphenyl has been determined by Attia et al. [23] using CHECKCELL program combined with the CRYSFIRE computer program. The investigation showed that p-quaterphenyl has a monoclinic structure with space group P21. In addition, the lattice parameters were found as: $a=8.11 \AA$, $b=$ $5.61 \AA, c=17.91 \AA$ and $\beta=95.8^{\circ}$. Fig. 2 illustrates the XRD patterns of the as-deposited and $\gamma$-irradiated $p$ quaterphenyl thin films. This figure shows that all diffraction peaks which arise from the thin films are attributed to $p$-quaterphenyl with the highest preferred orientation along the plane (004). After the irradiation process of $\mathrm{p}$-quaterphenyl thin film by $50 \mathrm{kGy}$ dose of gamma radiation, the peak intensity increases as shown in Fig. 2.

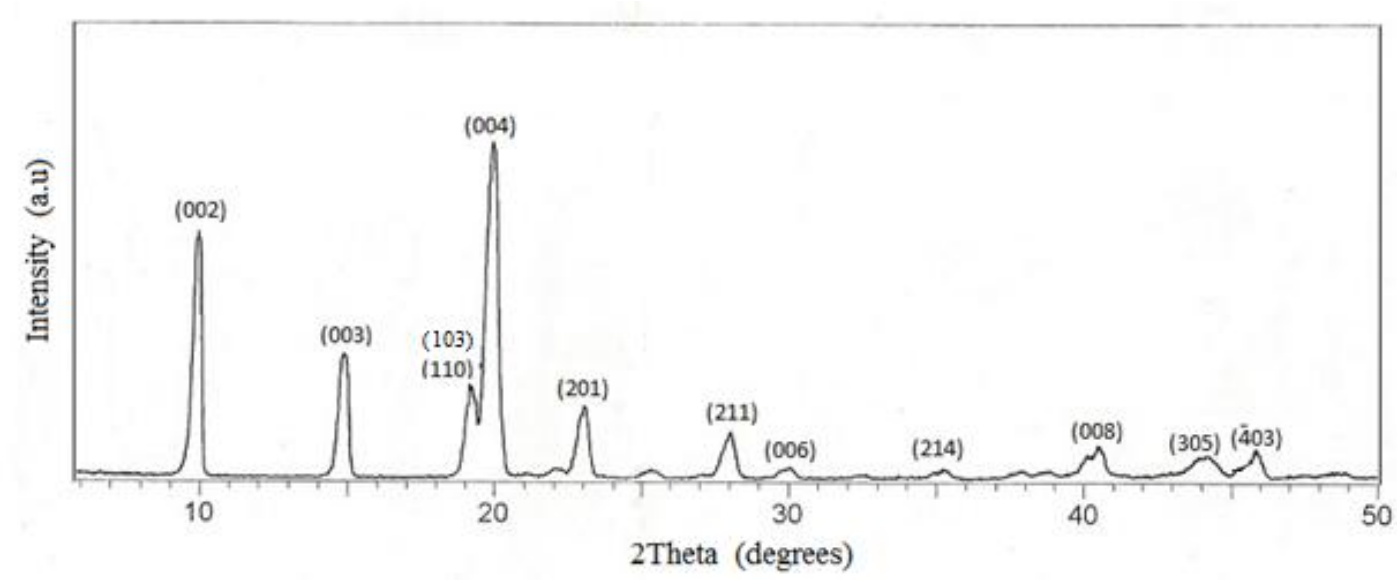

Fig. 1. XRD pattern of the $p$-quaterphenyl in powder form. 


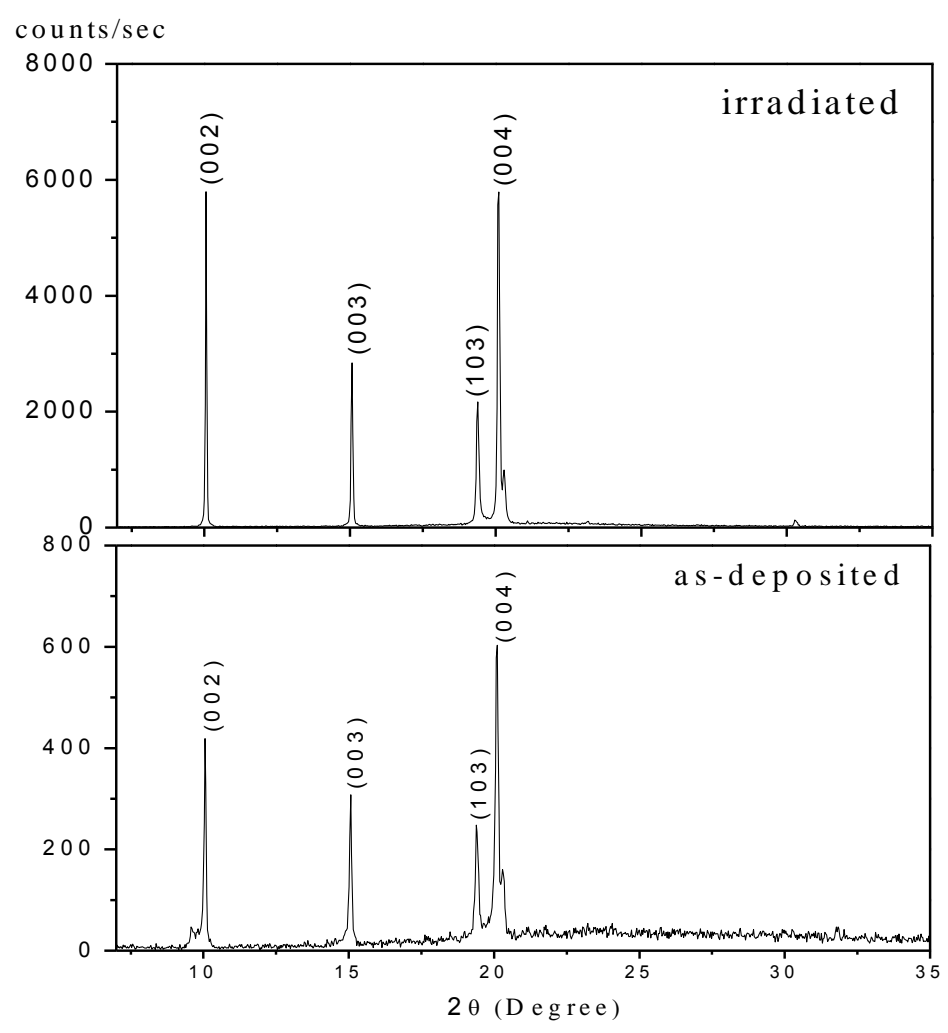

Fig. 2. XRD patterns of the as-deposited and $\gamma$-irradiated (50 kGy) p- quaterphenyl thin films.

Scherer's equation was used to calculate the crystallite size, D [24]:

$\mathrm{D}=\frac{0.9 \lambda}{\beta \cos \theta}$

Where $\lambda$ is the wavelength of the utilized X-ray, $\beta$ is the full width of a peak at half maximum intensity (FWHM) in radian, and $\theta$ is the reflection Bragg's angle. It was found that the average crystallite size of $p$-quaterphenyl thin film is increased from $83 \mathrm{~nm}$ to $119 \mathrm{~nm}$ after the irradiation process as listed in table 1 . This demonstrates that $50 \mathrm{kGy}$ dose of $\gamma$-irradiation improves the crystallinity of the films. The dislocation density, $\delta$, which is defined as the length of dislocation lines per unit volume and given as: $\delta=1 / \mathrm{D}^{2}[24]$, and the micro strain, $\varepsilon$, where $\varepsilon=\beta \cot \theta / 4$ [24], were calculated and inserted in table 1 as well. This table shows a decrease in both the dislocation density and the micro strain of $p$-quaterphenyl thin film after the irradiation process indicating the decrease in the disorder of films. These results indicate that gamma irradiation leads to changes in the microstructure of $p$-quaterphenyl thin films.

The morphology of film surface as well as the shape and size of $p$-quaterphenyl particles were determined by using field emission scanning electron microscope (FESEM). Fig. 3 shows FESEM images of the as-deposited and $\gamma$-irradiated $p$-quaterphenyl thin films, revealing rod-like particles formed with some agglomeration and aggregation. It is noticed that the rod-like particles maintained their shape even after the irradiation process with some changes in the length and width of the particles. The average length of these particles is ( $L=329$ $\mathrm{nm})$ and the average width is $(\mathrm{W}=180 \mathrm{~nm})$ with a ratio of $(\mathrm{L} / \mathrm{W}=1.83)$ for the as-deposited films, as can be seen in table 1 . While the values attributed to the $\gamma$-irradiated films are: $(L=500 \mathrm{~nm}),(\mathrm{W}=286 \mathrm{~nm})$, and $(\mathrm{L} / \mathrm{W}=1.75)$, listed in table 1 . It is obvious that the particle size was increased after $50 \mathrm{kGy}$ dose of gamma irradiation. These observations support the findings obtained from the X-ray diffraction. The higher values of the particle size compared with that of the crystallite size is due to that each particle is an agglomeration of several crystallites. 

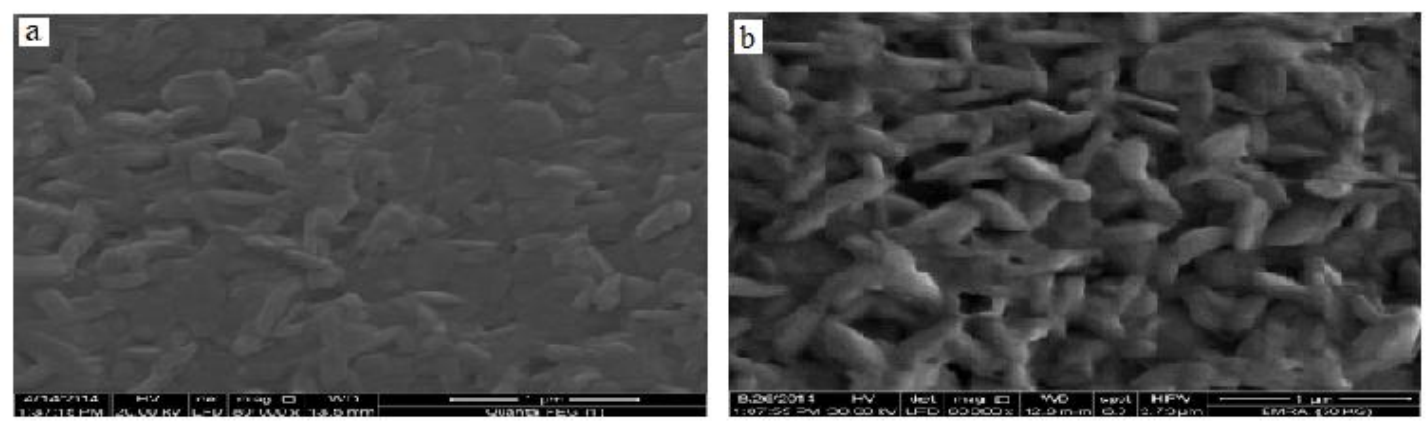

Fig. 3. FESEM images of (a) as-deposited and (b) $\gamma$-irradiated (50 kGy) p- quaterphenyl thin films.

Table 1. Structural data of the as-deposited and $\gamma$-irradiated (50 kGy) p- quaterphenyl thin films.

\begin{tabular}{ccccccc}
\hline & $\mathrm{D}(\mathrm{nm})$ & $\delta\left(\times 10^{-4} \mathrm{~nm}^{-2}\right)$ & $\varepsilon\left(\times 10^{-3}\right)$ & $\mathrm{L}(\mathrm{nm})$ & $\mathrm{W}(\mathrm{nm})$ & $\mathrm{L} / \mathrm{W}$ \\
\hline $\begin{array}{c}\text { As- } \\
\text { deposited }\end{array}$ & 83 & 1.47 & 3.21 & 329 & 180 & 1.83 \\
Irradiated & 119 & 0.71 & 2.25 & 500 & 286 & 1.75 \\
\hline
\end{tabular}

\subsection{Optical characterization}

The transmittance $T(\lambda)$ and the reflectance $R(\lambda)$ of the as-deposited and gamma irradiated (50 kGy dose) pquaterphenyl thin films of thickness $1148 \mathrm{~nm}$ in the wavelength range $(250-2500 \mathrm{~nm})$ are illustrated in Fig. 4. Investigation of this figure reveals that the absorption edge is in the wavelength range (500-850 nm), which separate the absorption region from the transparent region. Where the optical transition type and the corresponding value of energy gap can be determined from the absorption edge region. At transparent region of wavelength greater than $1200 \mathrm{~nm}$ where the extinction coefficient, $\mathrm{k}$, approaches to zero, the refractive index value, $n(\lambda)$, could be estimated for such films. Exposing the p-quaterphenyl thin film to $\gamma$-radiation dose of $50 \mathrm{kGy}$ shifts the transmittance spectrum toward the higher wavelength with significant reduction in the transmittance value. Such shift suggests decreasing in the optical band gap value of the $\gamma$-irradiated films. It is also observed that the gamma irradiation has no effect on the transmittance properties of as-deposited $p$ quaterphenyl thin film in the strong absorption region which extended to $500 \mathrm{~nm}$ of the spectrum.

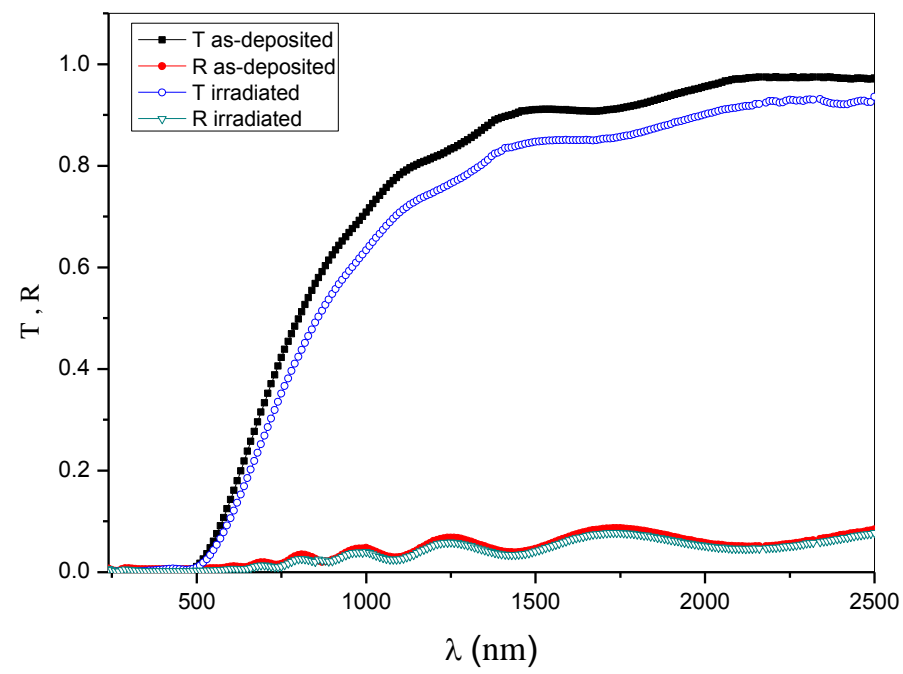

Fig. 4. The transmittance $T(\lambda)$ and the reflectance $R(\lambda)$ of the as-deposited and $\gamma$-irradiated (50 kGy) pquaterphenyl thin films of thickness $1148 \mathrm{~nm}$. 
The optical properties of p-quaterphenyl thin film can be studied by the complex refractive index, which composed of the refractive index $n(\lambda)$, as the real part, and the extinction coefficient $k(\lambda)$, as the imaginary part. The spectral dependence of the real part, $n(\lambda)$, for the as-deposited and $\gamma$-irradiated $p$-quaterphenyl thin films are presented in Fig. 5.

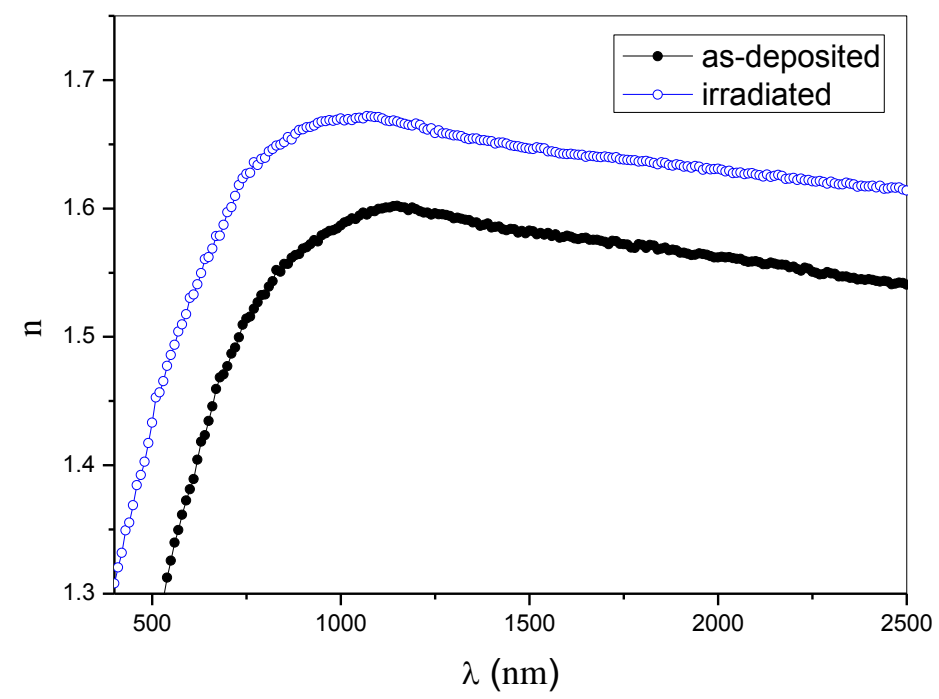

Fig. 5. The spectral dependence of the refractive index $n$ of the as-deposited and $\gamma$-irradiated (50 kGy) pquaterphenyl thin films.

The dispersion curves reveal an anomalous dispersion in the wavelength range (250-1200 $\mathrm{nm})$. While the region at longer wavelengths $(\lambda>1200 \mathrm{~nm})$ where $n(\lambda)$ decreases slowly, indicates the normal dispersion behavior of such films which is interpreted by the single oscillator model [25]. It is clear that the value of refractive index, $\mathrm{n}(\lambda)$, at any wavelength in the spectrum was increased after the $\gamma$-irradiation of 50 kGy dose which is due to changes in the structure of the film, as presented in Fig. 5 . The spectral distribution of the imaginary part, $\mathrm{k}(\lambda)$, for the as-deposited and $\gamma$-irradiated $\mathrm{p}$-quaterphenyl thin films are illustrated in Fig. 6.

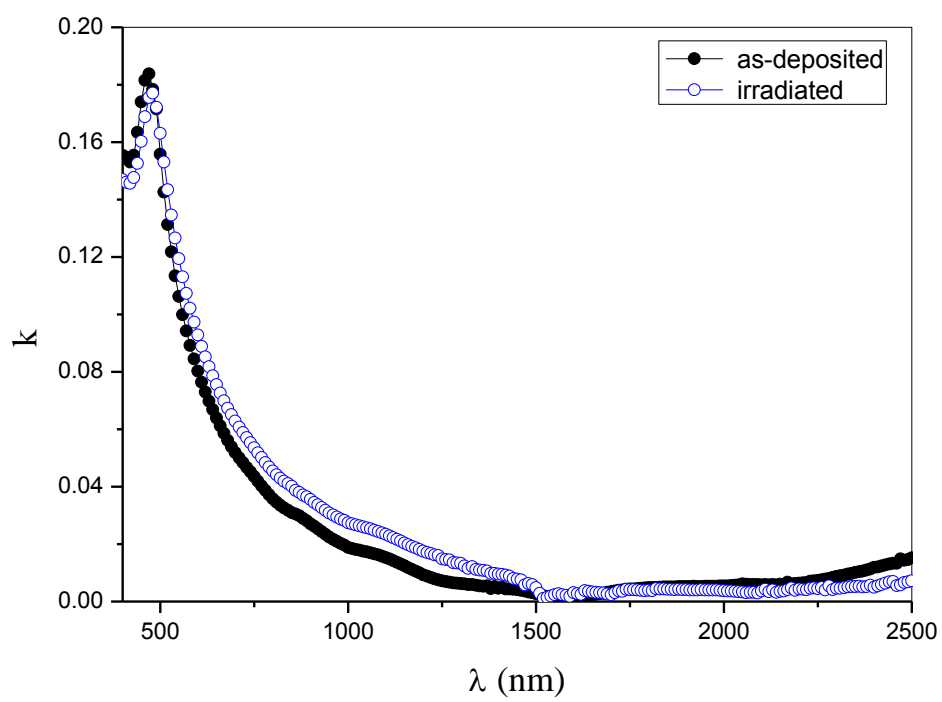

Fig. 6. The spectral behavior of the absorption index $k$ of the as-deposited and $\gamma$-irradiated (50 kGy) pquaterphenyl thin films. 
The extinction coefficient $k(\lambda)$ decreases with the increase of wavelength, reaching a cutoff value of $k$ at 1500 $\mathrm{nm}$. After the cutoff value, $\mathrm{k}$ begins to increase slightly with increasing wavelength indicating a free carrier absorption contribution [26]. Gamma irradiation with a dose of $50 \mathrm{kGy}$ gives rise to some increasing in the values of k, shifting the absorption edge toward higher-wavelength, with no change in the cutoff value.

From the analysis of the optical absorption near the absorption edge, the transition type and the optical band gap value $\mathrm{E}_{\mathrm{g}}$ can be estimated with the help of the following equation [27]:

$(\alpha h v)=B\left(h v-E_{g}\right)^{r}$

where $\mathrm{h} v$ is the photon energy, $\mathrm{B}$ is a characteristic constant independent of photon energy, and $\mathrm{r}=$ $1 / 2,3 / 2,2$, and 3 for direct allowed, direct forbidden, indirect allowed, and indirect forbidden transitions respectively. The best fit of the last equation was obtained for $(r=1 / 2)$ and presented in Fig. 7, indicating direct allowed transition. The intercept of the straight lines with the $x$-axis at $(\alpha h v=0)$ gives the optical band gap values for the as-deposited and $\gamma$-irradiated films, as can be seen in Fig. 7.

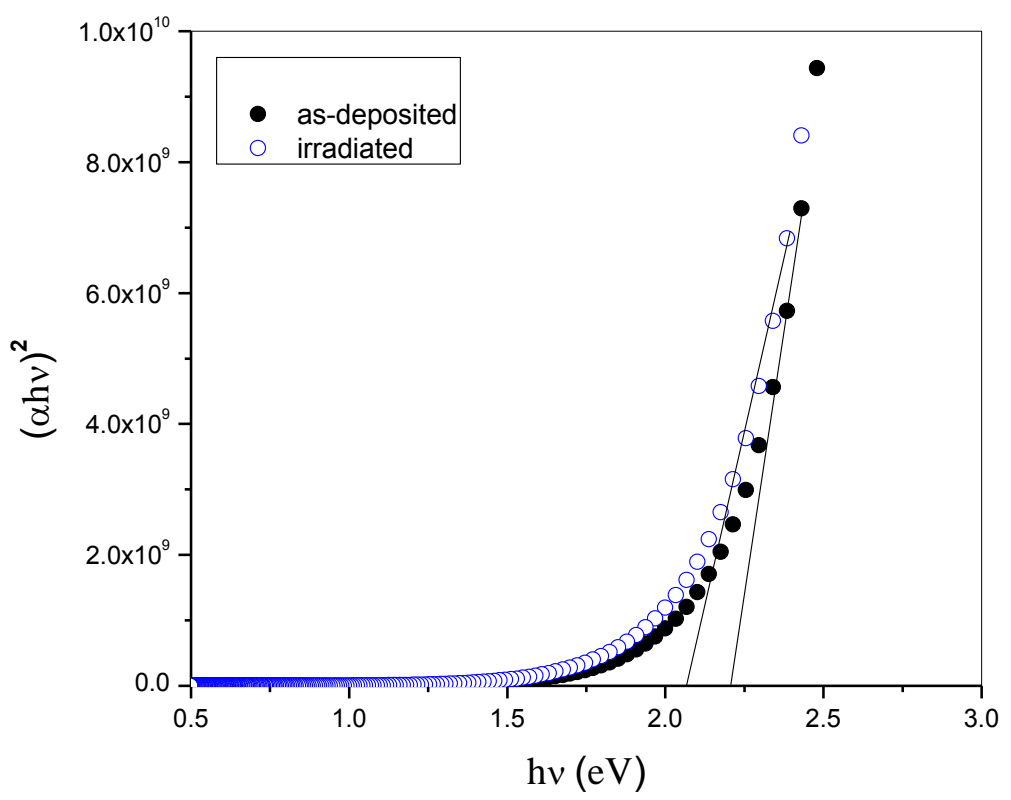

Fig. 7. Plot of $(\alpha h v)^{2}$ vs. (hv) of the as-deposited and $\gamma$-irradiated (50 kGy) p-quaterphenyl thin films.

The evaluation of the optical band gap values reveals some decrease from $2.2 \mathrm{eV}$ for the as-deposited film to $2.06 \mathrm{eV}$ for the irradiated film with $50 \mathrm{kGy}$ of gamma dose. These values are listed in table 2. This decrease in the optical band gap may be due to the adjustment in the particles aggregation in thin films as well as the increase in the particle size, which could be seen in Fig. 3. The optical band gap value obtained in this work agrees with the experimental data $(2.35 \mathrm{eV})$ [12] and the theoretical data $(2.20 \mathrm{eV})$ [28]. The spectral dependence of the absorption coefficient, $\alpha$, near the band edge, at the photon energy below the optical gap follows the Urbach's empirical formula [29]:

$\alpha=\alpha_{\mathrm{o}} \mathrm{e}^{\mathrm{hv} / \mathrm{E}_{\mathrm{U}}}$

where $E_{U}$ is the width of the band tail known as Urbach energy, and $\alpha_{\mathrm{o}}$ is a characteristic parameter of the material. The dependence of $\ln \alpha$ on hu for the as-deposited and $\gamma$-irradiated films are presented in Fig. 8. The Urbach energy value is obtained from the reciprocal of the slope of the linear portions of the graph of In $\alpha$ vs. hu. The estimated values of the Urbach energy are tabulated in table 2. It is noticed that the value of Urbach energy increases from $0.42 \mathrm{eV}$ to $0.47 \mathrm{eV}$ after the $\gamma$-irradiation of $50 \mathrm{kGy}$ dose for p-quaterphenyl thin films. 
This result supports our explanation of the findings of the optical band gap where the Urbach energy values change inversely with the optical band gap values. This means that the irradiation with $50 \mathrm{kGy}$ of gamma dose increases the influence of tailing on the band gap energy.

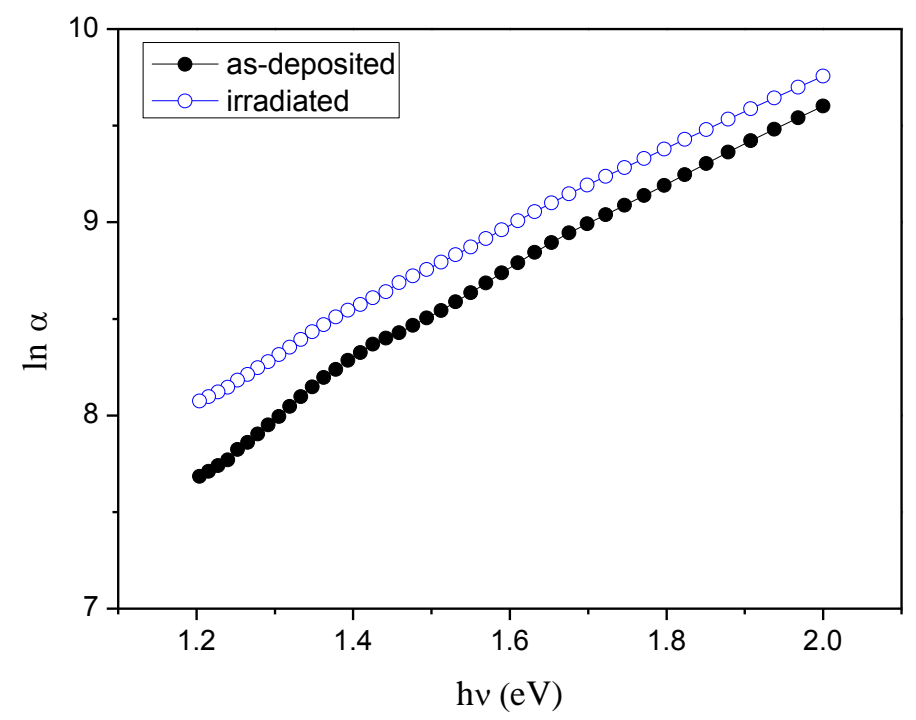

Fig. 8. The variation of ( $\ln \alpha$ ) vs. (hu) of the as-deposited and $\gamma$-irradiated (50 kGy) p-quaterphenyl thin films.

The complex dielectric constant $\varepsilon^{*}$ describes the interaction of the incident electromagnetic waves with the matter. The complex dielectric constant and its real $\varepsilon_{1}$ and imaginary $\varepsilon_{2}$ parts are determined by the following equations [30]:

$\varepsilon^{*}=\varepsilon_{1}+\mathrm{i} \varepsilon_{2}$

$\varepsilon_{1}=\mathrm{n}^{2}-\mathrm{k}^{2}$

$\varepsilon_{2}=2 \mathrm{nk}$

The real part of the dielectric constant $\varepsilon_{1}$ is mainly dependent on $\mathrm{n}^{2}$ due to the small value of $\mathrm{k}^{2}$. Hence, $\varepsilon_{1}$ describes how the material slows down the speed of light. While the imaginary part $\varepsilon_{2}$ is mainly dependent on $\mathrm{k}$ value, where $\mathrm{k}$ is related to the absorption coefficient. Consequently, $\varepsilon_{2}$ describes how the dielectric absorbs energy from an electric field owing to the dipole motion [31]. The variation of $\varepsilon_{1}$ and $\varepsilon_{2}$ depending on the photon energy hu for the as-deposited and $\gamma$-irradiated films is shown in Figs. 9 and 10, respectively. As observed that the values of $\varepsilon_{1}$ are higher than those of $\varepsilon_{2}$ in the studied range of hu. It is obvious that the values of both $\varepsilon_{1}(\mathrm{hv})$ and $\varepsilon_{2}(\mathrm{hv})$ were increased after the $\gamma$-irradiation of $50 \mathrm{kGy}$ dose which is due to the change in the structure of the film as mentioned before. 


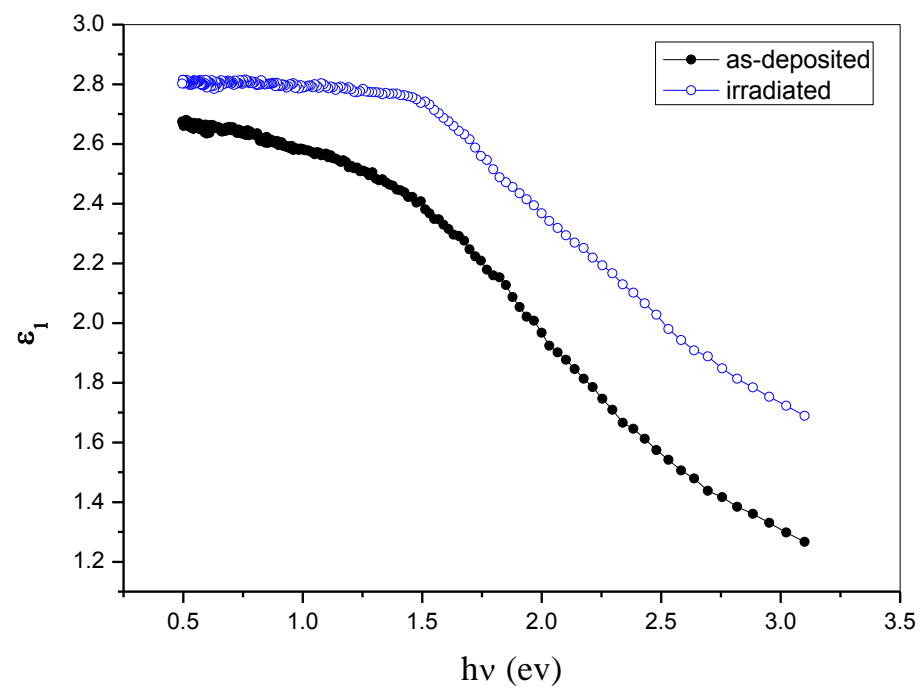

Fig. 9. The real part of the dielectric constant $\left(\varepsilon_{1}\right)$ vs. (hu) of the as-deposited and $\gamma$-irradiated (50 kGy) pquaterphenyl thin films.

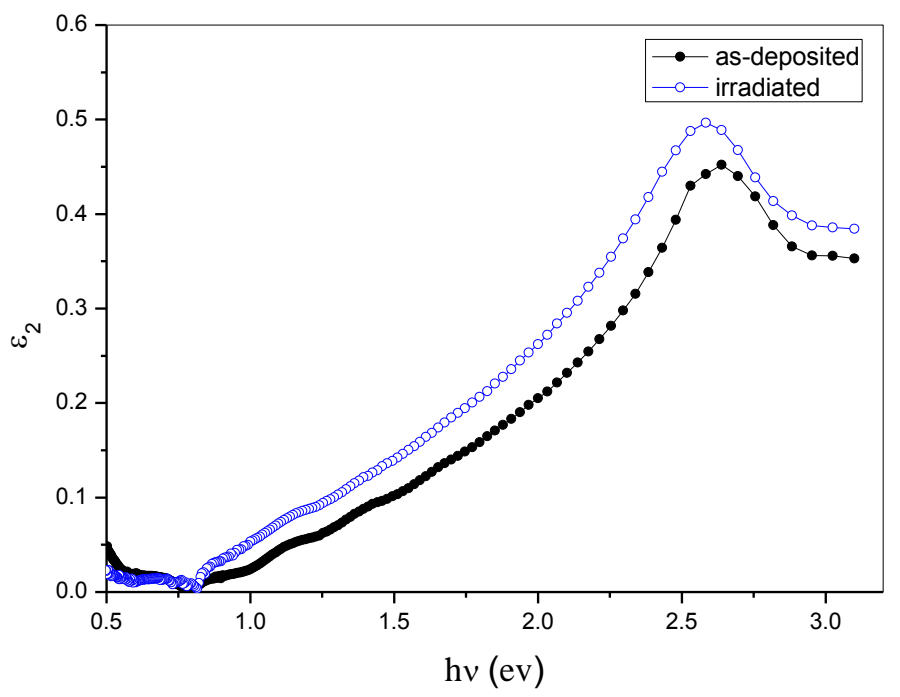

Fig. 10. The imaginary part of the dielectric constant $\left(\varepsilon_{2}\right)$ vs. (hu) of the as-deposited and $\gamma$-irradiated (50 kGy) p-quaterphenyl thin films.

Also, an absorption peak is observed at $2.64 \mathrm{eV}$ for the as-deposited film, which is shifted toward higherwavelength at $480 \mathrm{~nm}(2.58 \mathrm{eV})$ after the irradiation process, as illustrated in Fig. 10.

The normal dispersion behavior of $p$-quaterphenyl thin films, $n(\lambda)$, in the transparent region at wavelengths greater than $1200 \mathrm{~nm}$, can be analyzed using the single oscillator model which was expressed by Wimple and DiDomenico (WD-model) [25]:

$\left(n^{2}-1\right)=\frac{E_{d} E_{o}}{E_{0}^{2}-(h v)^{2}}$

Where $E_{o}$ is the single oscillator energy, and $E_{d}$ is the dispersion energy representing a measure of the strength of the electronic transitions. The variation of $\left(n^{2}-1\right)^{-1}$ depending on the square of the photon energy for the as-deposited and $\gamma$-irradiated films is illustrated in Fig. 11. 


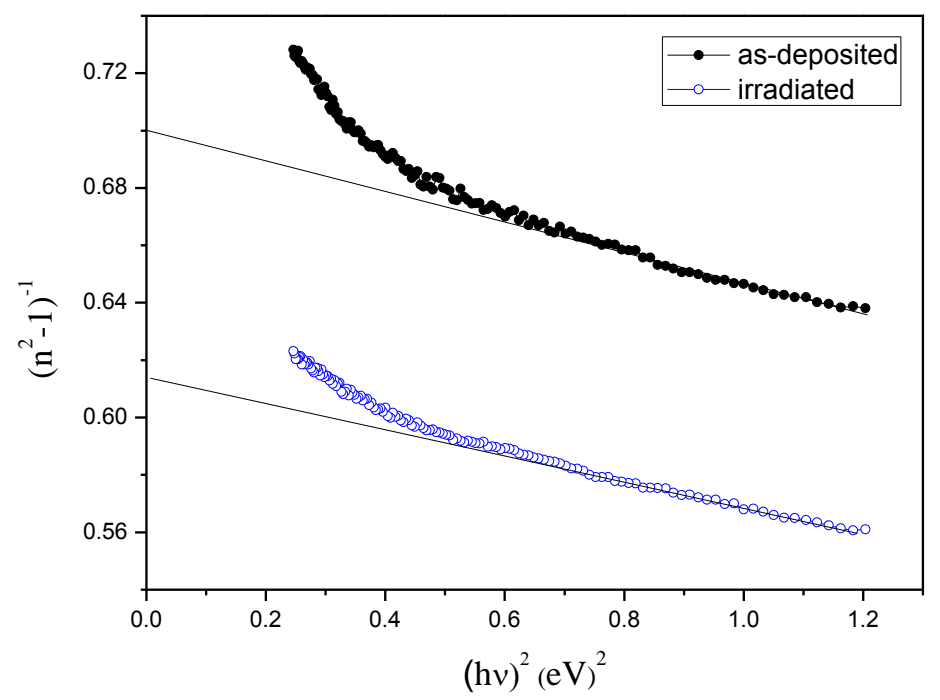

Fig. 11. Plot of $\left(n^{2}-1\right)^{-1}$ vs. (hu) $)^{2}$ of the as-deposited and $\gamma$-irradiated (50 kGy) p-quaterphenyl thin films.

This figure leads to obtain the dispersion parameters $E_{o}$ and $E_{d}$ as well as the high frequency dielectric constant $\varepsilon_{\infty}\left(\varepsilon_{\infty}=\mathrm{n}_{0}^{2}\right)$, by extrapolating the straight portion of each curve to intersect with the ordinate axis at (hv $\left.=0\right)$. The values of $\mathrm{E}_{0}, \mathrm{E}_{\mathrm{d}}, \mathrm{n}_{0}$, and $\varepsilon_{\infty}$, were inserted in table 2. Furthermore, the dispersion theory provides for the explanation of the spectral distribution of $\mathrm{n}^{2}$ versus $\lambda^{2}$ to deduce the lattice dielectric constant $\varepsilon_{\mathrm{L}}$, by a procedure characterizes the free carriers contribution and the lattice vibration modes of the dispersion. The relation between $\mathrm{n}^{2}$ and $\lambda^{2}$ in the transparent region is given as [32]:

$\mathrm{n}^{2}=\varepsilon_{\mathrm{L}}-\frac{\mathrm{e}^{2}}{4 \pi^{2} \varepsilon_{0} \mathrm{c}^{2}} \frac{\mathrm{N}}{\mathrm{m}^{*}} \lambda^{2}$

where $e$ is the electronic charge, $\varepsilon_{0}$ is the permittivity of free space, $c$ is the speed of light and $N / \mathrm{m}^{*}$ is the ratio of free carrier concentration to the effective mass. Fig. 12 reveals the variation of $n^{2}$ versus $\lambda^{2}$ for the asdeposited and $\gamma$-irradiated $p$-quaterphenyl thin films.

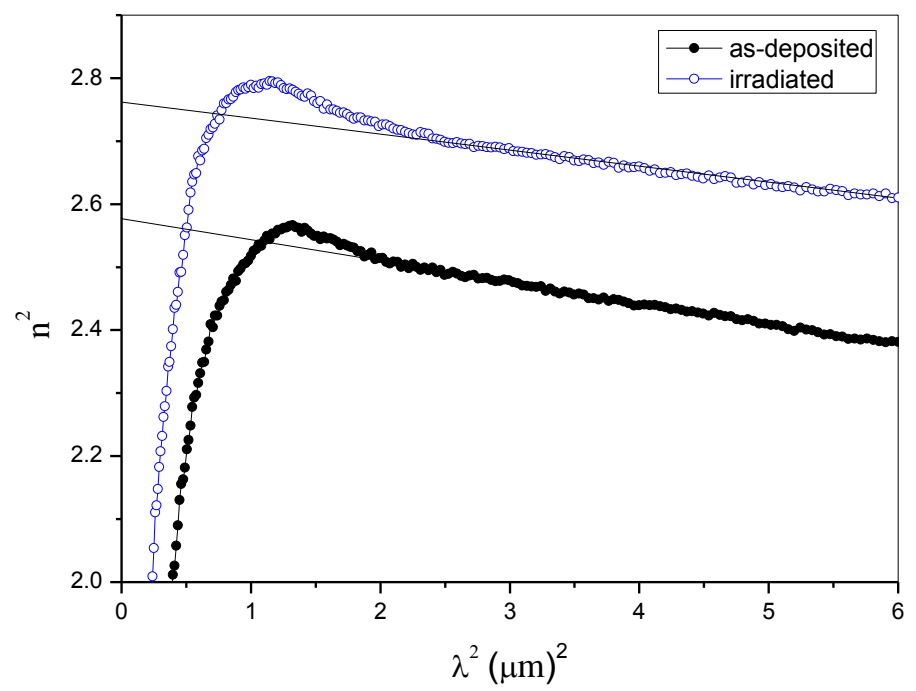

Fig. 12. Plot of $n^{2}$ vs. $\lambda^{2}$ of the as-deposited and $\gamma$-irradiated (50 kGy) p-quaterphenyl thin films. 
The dependence of $n^{2}$ on $\lambda^{2}$ is linear at the longer wavelengths. The values of $\varepsilon_{L}$ were obtained by extrapolating these linear parts of each curve to intersect with the ordinate axis at zero wavelength. While the values of $\mathrm{N} / \mathrm{m}^{*}$ were estimated from the slope of these linear parts illustrated in Fig. 12. The values of $\varepsilon_{\mathrm{L}}$ and $\mathrm{N} / \mathrm{m}^{*}$ were inserted in the table 2 . It is observed that the values of $\varepsilon_{\mathrm{L}}$ are greater than those of $\varepsilon_{\infty}$. This may be due to the lattice vibrations and free carriers contribution [33]. Also, the plasma frequency, $\omega_{\mathrm{P}}$, can be deduced according to the Drude model, via the relationship between $\omega_{\mathrm{P}}$ and the ratio $\mathrm{N} / \mathrm{m}^{*}$ [34]:

$$
\omega_{\mathrm{P}}=\left(\frac{\mathrm{e}^{2}}{\varepsilon_{\mathrm{o}} \varepsilon_{\mathrm{L}}} \frac{\mathrm{N}}{\mathrm{m}^{*}}\right)^{1 / 2}
$$

The values of $\omega_{\mathrm{P}}$ were calculated and inserted in table 2 .

Table 2. Optical parameters of the as-deposited and $\gamma$-irradiated (50 kGy) p-quaterphenyl thin films.

\begin{tabular}{|c|c|c|c|c|c|c|c|c|c|}
\hline & $\begin{array}{l}E_{g} \\
(e V)\end{array}$ & $\begin{array}{c}E_{U} \\
(e V)\end{array}$ & $\begin{array}{l}E_{0} \\
(e V)\end{array}$ & $\begin{array}{c}E_{d} \\
(e V)\end{array}$ & $\mathrm{n}_{\mathrm{o}}$ & $\varepsilon_{\infty}$ & $\varepsilon_{\mathrm{L}}$ & $\begin{array}{c}\mathrm{N} / \mathrm{m}^{*} \\
\left(10^{55} \mathrm{~kg}^{-1} \mathrm{~m}^{-3}\right)\end{array}$ & $\begin{array}{c}\omega_{\mathrm{p}} \\
\left(10^{14} \mathrm{rad} / \mathrm{s}\right)\end{array}$ \\
\hline $\begin{array}{c}\text { As- } \\
\text { deposited }\end{array}$ & 2.20 & 0.42 & 4.76 & 7.00 & 1.56 & 2.43 & 2.58 & 4.15 & 2.16 \\
\hline Irradiated & 2.06 & 0.47 & 3.91 & 6.40 & 1.62 & 2.63 & 2.76 & 3.17 & 1.82 \\
\hline
\end{tabular}

The modeling analysis of the nature of the nonlinear optical parameters denotes that the linear refractive index, $\mathrm{n}$, and the optical band gap, Eg, of the solids are very significant parameters to explain the optical nonlinearity [35]. The nonlinear absorption coefficient, $\beta_{c}$, can be calculated using the equation [36]:

$\beta_{c}=\frac{k_{c} E_{p}^{1 / 2} F}{n^{2} E_{g}^{3}}$

where $k_{c}$ is a material independent constant, which has a value of 1940 in units such that $\beta_{c}$ is in $\mathrm{cm} / \mathrm{GW}$ and $\mathrm{Eg}$ in $\mathrm{eV} . \mathrm{E}_{\mathrm{p}}$ is related to the Kane momentum parameter and has a value of about $21 \mathrm{eV}$ for direct-gap semiconductors. These values are agreeable for most semiconductors [37, 38]. F is a function explains the dispersion of $\beta_{c}$ versus hu. This function is dependent on the band structure and determines the states of energy that are coupled. $\mathrm{F}$ can be estimated using the following equation $[39,40]$ :

$\mathrm{F}=\frac{\left(\frac{2 \mathrm{~h} v}{\mathrm{Eg}}-1\right)^{3 / 2}}{\left(\frac{2 \mathrm{~h} v}{\mathrm{Eg}}\right)^{5}}$

The non-linear absorption coefficient spectra for the as-deposited and $\gamma$-irradiated $p$-quaterphenyl thin films are shown in Fig. 13. 


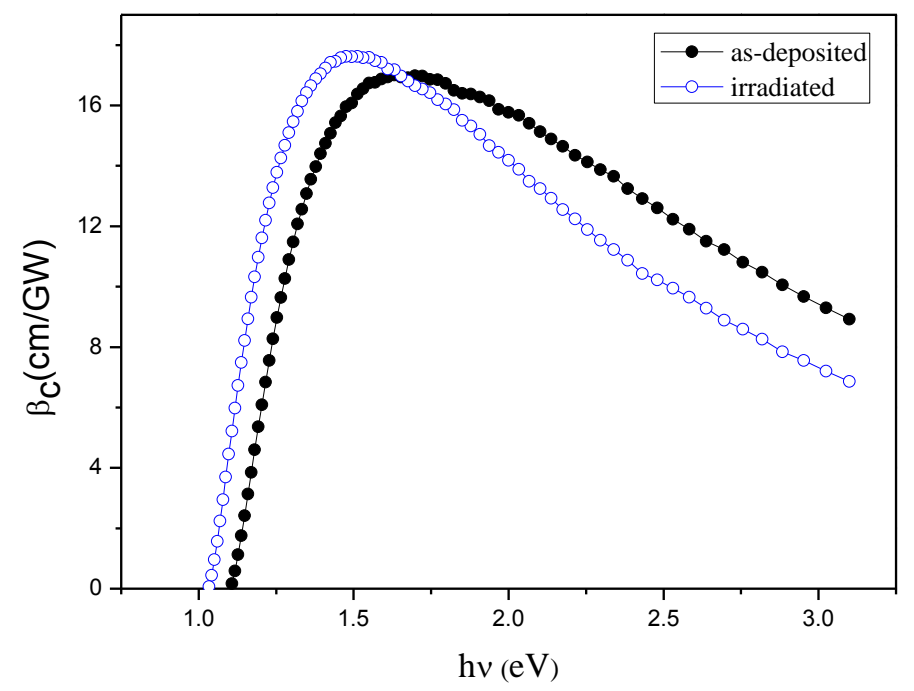

Fig. 13. The non-linear absorption coefficient spectra of the as-deposited and $\gamma$-irradiated (50 kGy) pquaterphenyl thin films.

This figure shows that the maximum value of $\beta_{\mathrm{c}}(17.01 \mathrm{~cm} / \mathrm{GW})$ was increased to the value of $17.62 \mathrm{~cm} / \mathrm{GW}$ after the irradiation with $50 \mathrm{kGy}$ of gamma dose. Also, the energy corresponding to the maximum value of $\beta_{\mathrm{c}}$ at $1.63 \mathrm{eV}(760 \mathrm{~nm})$ was shifted toward the higher wavelength at $820 \mathrm{~nm}(1.51 \mathrm{eV})$ after the irradiation process, as can be seen in Fig. 13. It is of interest to note that, the ratio of the optical band gap, Eg, to the energy corresponding to the maximum value of the nonlinear absorption coefficient for both the as-deposited and $\gamma$-irradiated films, is equal to $\sim 1$.4. Such energy ratio has been examined with other various reported materials [35], which found an excellent coincidence with the obtained ratio, (i.e. 1.4).

\section{Conclusions}

p-quaterphenyl thin films were deposited utilizing thermal evaporation method. The effects of the exposure to gamma radiation of Cobalt-60 radioactive source with a dose of $50 \mathrm{kGy}$, on the structural and optical properties of $p$-quaterphenyl thin films have been studied. The XRD results showed that the average crystallite size was increased from $83 \mathrm{~nm}$ to $119 \mathrm{~nm}$ after exposing film to gamma ray, indicating an improvement in the crystallinity of the thin films. While the dislocation density and the micro strain of films were decreased after the irradiation process indicating the decrease in the disorder of films. The surface morphology of the asdeposited and $\gamma$-irradiated films revealed rod-like particles formed with some agglomeration and aggregation. The particle size was increased after $50 \mathrm{kGy}$ dose of $\gamma$-irradiation. These observations suggest that $\gamma$-irradiation with $50 \mathrm{kGy}$ dose gives rise to changes in the microstructure of the p-quaterphenyl thin films. The refractive and absorption indices of the as-deposited and $\gamma$-irradiated p-quaterphenyl thin films were determined from the transmission and reflection data in the wavelength range from 250 to $2500 \mathrm{~nm}$. The spectral behavior of the absorption index, $\mathrm{k}$, revealed an absorption peak at $470 \mathrm{~nm}$ and free carriers absorption at $\lambda>1500 \mathrm{~nm}$. Gamma irradiation gives rise to shift the absorption peak position toward higher wavelength at $480 \mathrm{~nm}$ with no change in the cutoff value of $k$, at $1500 \mathrm{~nm}$. The transition type for the as-deposited and $\gamma$-irradiated $p$ quaterphenyl thin films was found to be direct allowed transition. The optical band gap value of the asdeposited films was obtained to be $2.2 \mathrm{eV}$, which decreased to $2.06 \mathrm{eV}$ after the exposure of films to gamma irradiation. The values of the obtained Urbach energy were found to change inversely with the optical band gap values which means that the $\gamma$-irradiation increases the influence of tailing on the band gap energy. The normal dispersion of the refractive index was explained using the single oscillator model together with the Drude model to distinguish between the bound and free carriers contribution. It was found that the values of the lattice dielectric constant $\varepsilon_{\mathrm{L}}$ are greater than those of the high frequency dielectric constant $\varepsilon_{\infty}$, which may be due to the lattice vibrations and free carriers contribution. Also, the values of the dispersion parameters of 
p-quaterphenyl thin films were affected by $\gamma$-irradiation of $50 \mathrm{kGy}$ dose. The nonlinear optical studies showed that the maximum value of non-linear absorption coefficient, $\beta_{c}$, of $p$-quaterphenyl thin films was increased to the value of $17.62 \mathrm{~cm} / \mathrm{GW}$ and its corresponding energy shifted toward the higher wavelength at $820 \mathrm{~nm}$ after the irradiation process.

\section{References}

[1] S.E. Al Garni, A.A.A. Darwish, Solar Energy Materials and Solar Cells 160 (2017) 335.

[2] S. Rasouli, S.J. Moeen, J. Alloys Compd. 509 (2011) 1915.

[3] D. Gupta, Y. Hong, Org. Electron. 11 (2010) 127.

[4] A. Natalia Azarova, J.W. Owen, C.A. McLellan, M.A. Grimminger, E.K. Chapman, J.E. Anthony, O.D. Jurchescu, Org. Electron. 11 (2010) 1960.

[5] S. Nenon, D. Kanehira, N. Yoshimoto, F. Fages, C. Videlot-Ackermann, Thin Solid Films 518 (2010) 5593.

[6] M.M. El-Nahass, H.M. Zeyada, K.F. Abd-El-Rahman, A.A.M. Farag, A.A.A. Darwish, Solar Energy Materials and Solar Cells 91 (2007) 1120.

[7] M.M. El-Nahass, K.F. Abd El-Rahman, A.A. M. Farag, A.A.A. Darwish, Organic Electronics 6 (2005) 129.

[8] D.J. Gundlach, Y.Y. Lin, T.N. Jackson, D.G. Schlom, Appl. Phys. Lett. 71 (1997) 3853.

[9] M. Era, T. Tsutsui, S. Saito, Appl. Phys. Lett. 67 (1995) 2436.

[10] S. Tasch, C. Brandstaetter, F. Meghdadi, G. Leising, G. Froyer, L. Athouel, Adv. Mater. 9 (1997) 33.

[11] A.A. Attia, M.M. Saadeldin, H.S. Soliman, A.-S. Gadallah, K. Sawaby, Optical Materials 62 (2016) 711.

[12] A.A.A. Darwish, Infrared Phys. Technol. 82 (2017) 96.

[13] A. M. Ibrahim, L. I. Soliman, Rad. Phys. \& Chem. 53 (1998) 469.

[14] E. Atanassova, A. Pasksleva, R. Konakova, D. Spassov, V. F. Mitin, Microelectronics J. 32 (2001) 553.

[15] R. L. Clough, Nucl. Instr. and Meth. in Phys. Res. B185 (2001) 8.

[16] E. Colby, G. Lum, T. Plettner, J. Spencer, IEEE Trans. Nucl. Sci. NS-49 (2002) 2857.

[17] K. Arshak, A. Arshak, S. Zleetni, O. Korostynska, IEEE Trans. Nucl. Sci. NS-51 (2004) 2250.

[18] K. Arshak, O. Korostynska, J. Molly, J. Harris, IEEE Sensors J. 6 (2006) 656.

[19] K. Arshak, O. Korostynska, Mater. Sci. Eng. B133 (2006) 1.

[20] S. Tolansky, Multiple-Beam Interference Microscopy of Metals, Academic Press, London, 1970, p. 55.

[21] L.A. Agiev, I.N. Shklyarevskii, J. Prekel Spekt 76 (1978) 380.

[22] M. Di Giulio, G. Micocci, R. Rella, P. Siciliano, A. Tepore, Phys Status Solidi A 136 (1993) K101.

[23] A.A. Attia, H.S. Soliman, M.M. Saadeldin, K. Sawaby, Synthetic Metals 205 (2015) 139.

[24] S. Anwar, M. Pattanaik, B.K. Mishra, S. Anwar, Mat. Sci. Semicon. Proc. 34 (2015) 45.

[25] S.H. Wemple, M. Di Domenico, Phys. Rev. B 3 (1971) 1338. 
[26] H.M. Zeyada, M.M. EL-Nahass, I.S. Elashmawi, A.A. Habashi, J. Non-Cryst. Solids 358 (2012) 625.

[27] J. Bardeen, F.J. Blatt, L. Hall, in: R.G. Breckenridge, B.R. Russel, E.E. Hahn (Eds.), Proceedings of Photoconductivity Conference, Wiley, New York, 1956, p. 146.

[28] P. Puschnig, C.A. Draxl, Phys. Rev. B 60 (1999) 7891.

[29] A. Elshafie, B.A. Mansour, A. Abdel-All, J. Phys. Chem. Solids 60 (1999) 483.

[30] M.M. El-Nahass, H.M. Abd El-Khalek, A.M. Nawar, Opt. Commun. 285 (2012) 1872.

[31] T.E. Youssef, M.M. El-Nahass, E.F.M. El-Zaidia, J. Lumin. 138 (2013) 187.

[32] M.M. El- Nahass, M. Dongol, M. Abou-zied, A. El-Denglawey, Physica B 368 (2005) 179.

[33] Konstantinov, T. Babeva, S. Kitova, Appl. Opt. 37 (1998) 4260.

[34] H.M. Zeyada, M.M. EL-Nahass, S.A. Samak, Journal of Non-Crystalline Solids 358 (2012) 915.

[35] F.A. Abdel-Wahab, F. El-Diasty, M. Abdel-Baki, Physics letters A 373 (2009) 3855.

[36] E. W. Van Stryland, M. A. Woodall, H. Vanherzeele, M. J. Soileau, Opt. Lett. 10 (1985) 490.

[37] M. Sheik-Bahae, D.C. Hutchings, D.J. Hagan, E.W. Van Stryland, IEEE J. Quantum Electron. 27 (1991) 1296.

[38] H. Garcia, R. Kalyanaraman, J. Phys. B: At. Mol. Opt. Phys. 39 (2006) 2737.

[39] M. H. Weiler, Solid State Commun. 39 (1981) 937.

[40] B. S. Wherrett, J. Opt. Soc. Am. B 1 (1984) 67. 